\title{
Cerebellar ataxia associated with anti-glutamic acid decarboxylase (anti-GAD) autoantibodies: a rare and puzzling disease
}

\author{
Ataxia cerebelar associada com anticorpos anti-GAD: uma rara e enigmática doença \\ Hélio A. G. Teive
}

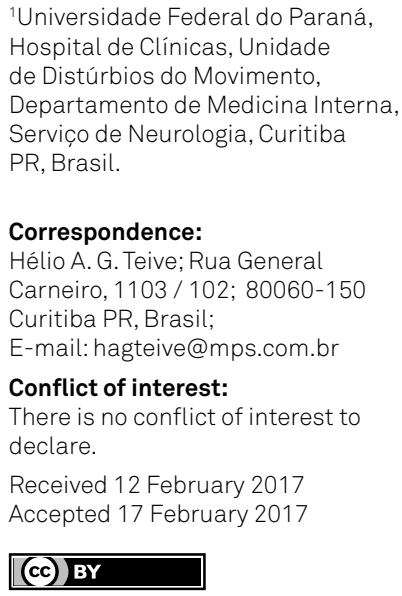

'Universidade Federal do Paraná, Hospital de Clínicas, Unidade de Distúrbios do Movimento, Departamento de Medicina Interna, Serviço de Neurologia, Curitiba PR, Brasil.

Correspondence:

Hélio A. G. Teive; Rua General Carneiro, 1103 / 102; 80060-150

Curitiba PR, Brasil;

E-mail:hagteive@mps.com.br

Conflict of interest:

There is no conflict of interest to declare.

Received 12 February 2017

Accepted 17 February 2017

(c) BY

$\mathrm{N}$ eurological disorders associated with anti-glutamic acid decarboxylase autoantibodies (anti-GAD Abs) include stiff-person syndrome (SPS) and its variants (stiff-trunk syndrome, stiff-limb syndrome, progressive encephalomyelitis with rigidity and myoclonus [PERM], or "SPS plus", and paraneoplastic SPS) as well as epilepsy, abnormal eye movements, limbic encephalitis and cerebellar ataxia ${ }^{1,2}$. These syndromes are often found in association with other autoimmune disorders, such as type 1 diabetes mellitus, Addison's disease, thyroiditis, vitiligo, hemolytic anemia, myasthenia gravis, vitamin B12 deficiency due to autoimmune destruction of parietal cells (pernicious anemia) and polyglandular autoimmune syndromes ${ }^{1,2,3,4}$. GAD is a cytoplasmic enzyme that produces GABA $(\gamma$-aminobutyric acid), the main inhibitory neurotransmitter in the central nervous system (CNS). It is expressed predominantly in CNS GABAergic neurons and pancreatic islet $\beta$-cells and has two isoforms, GAD 65, which is more involved in neurological diseases, and GAD 67 $7^{1,2,3,4}$ GAD is also found in the liver, kidneys, adrenal glands, ovaries and testes, but in lower concentrations. Anti-GAD Abs inhibit GAD activity and synthesis of GABA ${ }^{1,2,3,4}$. Anti-GAD 65 autoantibodies were described initially in type 1 diabetes mellitus patients and are considered a biomarker for this disease ${ }^{3}$. In 1956, Moersch and Woltman ${ }^{5}$ published a case report of a 49-year-old man with progressive fluctuating muscular rigidity and spasm ("stiff-man syndrome") along with observations of thirteen other cases. More recently, the term "stiff-man syndrome" was replaced by "stiff-person syndrome" (SPS), and anti-GAD autoantibodies were found to be associated with this disorder and its variants ${ }^{4}$. Immune-mediated cerebellar ataxias include gluten ataxia, paraneoplastic cerebellar degeneration, ataxia associated with systemic lupus erythematosus, Hashimoto's encephalopathy (cerebellar type), primary autoimmune cerebellar ataxia and cerebellar ataxia associated with GAD autoantibodies (GAD Abs-CA) ${ }^{4}$. This last condition was described in 1997 by Saiz et al. ${ }^{6}$ in three patients who also had late-onset insulin-dependent diabetes mellitus and polyendocrine autoimmunity. Since then, several case reports and a study of a small series of patients have been published ${ }^{2,7,8}$, as well as a study of fourteen patients by Honnorat et al. ${ }^{9}$ published in 2001. GAD Abs-CA occurs most commonly in women in their sixth decade, and the prognosis is usually poor ${ }^{9,10}$. In 2008, Hadjivassiliou et al.11 published a prospective study on the prevalence of immune-mediated cerebellar ataxia. Of 400 patients with progressive cerebellar ataxia, $23 \%$ had idiopathic sporadic ataxia. The prevalence of autoimmune disease in this group was $47 \%$. Gluten ataxia was diagnosed in $27 \%$ of the patients, paraneoplastic cerebellar degeneration (PCD) in $3 \%$ and GAD Abs-CA in $2 \%{ }^{11}$ In this issue of Arquivos de Neuro-Psiquiatria, Aguiar et al. ${ }^{12}$ describe the clinical characteristics of patients with GAD Abs-CA. In their retrospective study they evaluate three patients with this condition (one female and two males, mean age 49.3 years), two of whom also had SPS. All the patients had high GAD-Ab titers (> $100 \mathrm{IU} / \mathrm{mL}$ ) without any paraneoplastic neurological syndromes. After treatment with intravenous immunoglobulin, one patient made a partial recovery but the two others showed no improvement.12 Associated endocrine disease included latent autoimmune diabetes in adults (LADA) in two patients, and autoimmune polyglandular endocrinopathy type 2, or Schmidt's syndrome in one patient ${ }^{12}$. In 2016, Mitoma et al. ${ }^{10}$ published a review of guidelines for treatment of immune-mediated cerebellar ataxias. They concluded that although various types of immunotherapies (including intravenous methylprednisolone, intravenous immunoglobulins, plasma exchange and rituximab) 
have been used in patients with GAD Abs-CA, most patients continue to have significant disability and a poor prognosis. ${ }^{10}$ However, in clinical practice some patients with this disorder show an improvement in the cerebellar ataxia symptoms ${ }^{2,8,9}$.
Despite the controversy surrounding treatment of this condition and its prognosis, it is now imperative that neurologists be able to recognize this form of auto-immune cerebellar ataxia caused by anti-GAD Abs.

\section{References}

1. Saiz A, Blanco Y, Sabater L, González F, Bataller L, Casamitjana R et al. Spectrum of neurological syndromes associated with glutamic acid decarboxylase antibodies: diagnostic clues for this association. Brain. 2008;131(10):2553-63. https://doi.org/10.1093/brain/awn183

2. Fernandes M, Munhoz RP, Carrilho PE, Arruda WO, Lorenzoni PJ, Scola RH et al. Neurological disorders associated with glutamic acid decarboxylase antibodies: a Brazilian series. Arq Neuropsiquiatr. 2012;70(9):657-61. https://doi.org/10.1590/S0004-282X2012000900002

3. Mitoma H, Adhikari K, Aeschlimann D, Chattopadhyay P, Hadjivassiliou M, Hampe CS et al. Consensus paper: neuroimmune mechanisms of cerebellar ataxias. Cerebellum. 2016;15(2):213-32. https://doi.org/10.1007/s12311-015-0664-x

4. Sarva H, Deik A, Ullah A, Severt WL. Clinical spectrum of stiff person syndrome: A review of recent reports. Tremor Other Hyperkinet Mov (N Y). 2016;6:340. . https://doi.org/10.7916/D85M65GD

5. Moersch FP, Woltman HW. Progressive fluctuating muscular rigidity and spasm ("stiff-man" syndrome): report of a case and some observations in 13 other cases. Proc Staff Meet Mayo Clin. 1956;25:421-7.

6. Saiz A, Arpa J, Sagasta A, Casamitjana R, Zarranz JJ, Tolosa E et al. Autoantibodies to glutamic acid decarboxylase in three patients with cerebellar ataxia, late-onset insulin diabetes mellitus, and polyendocrine autoimmunity. Neurology. 1997;49(4):1026-30. https://doi.org/10.1212/WNL.49.4.1026
7. Abele M, Weller M, Mescheriakov S, Bürk K, Dichgans J, Klockgether T. Cerebellar ataxia with glutamic acid decarboxylase autoantibodies. Neurology. 1999;52(4):857-9. https://doi.org/10.1212/WNL.52.4.857

8. Pedroso JL, Braga-Neto P, Dutra LA, Barsottini OG. Cerebellar ataxia associated to anti-glutamic acid decarboxylase autoantibody (anti-GAD): partial improvement with intravenous immunoglobulin therapy. Arq Neuropsiquiatr. 2011;69(6):993. https://doi.org/10.1590/S0004-282X2011000700030

9. Honnorat J, Saiz A, Giometto B, Vincent A, Brieva L, Andres C et al. Cerebellar ataxia with anti-glutamic acid decarboxylase antibodies: study of 14 patients. Arch Neurol. 2001;58(2):225-30. https://doi.org/10.1001/archneur.58.2.225

10. Mitoma H, Hadjivassiliou M, Honnorat J. Guidelines for treatment of immune-mediated cerebellar ataxias. Cerebellum Ataxias. 2015;2(1):14. https://doi.org/10.1186/s40673-015-0034-y

11. Hadjivassiliou M, Boscolo S, Tongiorgi E, Grünewald RA, Sharrack B, Sanders DS et al. Cerebellar ataxia as a possible organ-specific autoimmune disease. Mov Disord. 2008;23(10):1270-7. https://doi.org/10.1002/mds.22129

12. Aguiar T, Fragoso A, Albuquerque CR, et al. Clinical characteristics of patients with cerebellar ataxia associated with anti-GAD antibodies. Arq Neuropsiquiatr 2017;75(3):142-46. https://doi.org/10.1590/0004-282X20170011 